\title{
Les Algues Indicatrices De L'eutrophisation Et De La Contamination De La Lagune De Korba (Cap Bon Tunisie)
}

\author{
Rahmouni R . \\ Bouden $S$. \\ Oueslati W. \\ Sbei A. \\ Chaabani F.
}

Laboratoire des Ressources Minérales et Environnement

Faculté des Sciences de Tunis, Université de Tunis El Manar

doi: 10.19044/esj.2016.v12n17p149 URL:http://dx.doi.org/10.19044/esj.2016.v12n17p149

\begin{abstract}
The concentrations of heavy metals ( $\mathrm{Fe}, \mathrm{Zn}, \mathrm{Pb}$ and $\mathrm{Mn}$ ) were determined in two green algae (Enteromorpha intestinalis and Chaetomorpha Linium) collected from April 2012 to February 2013 in nine stations characterized by the abundance of these algae throughout the year. Determination of $\mathrm{Pb}, \mathrm{Mn}$ and $\mathrm{Zn}$ was carried out by graphite furnace atomic absorption spectrometer while Fe by flame atomic absorption spectrometer (using the Thermo Scientific ICE 3300 AA Spectrometer). An evaluation of the degree of eutrophication is made through the physicochemical study of 31 samples in the surface water. The results underline that Korba is an eutrophic lagoon. The heavy metal concentrations in the water samples decreased in the sequence of $\mathrm{Fe}>\mathrm{Zn}>\mathrm{Mn}>\mathrm{Pb}$, while in Chaetomorpha Linium, Enteromorpha intestinalis and sediment exhibited the same pattern across the sites: $\mathrm{Fe}>\mathrm{Mn}>\mathrm{Zn}>\mathrm{Pb}$. The results show that metal accumulation capacity in both macro-algae varies according to the season and depends on the species. By comparison with other studies in the world, the levels found in the algae of Korba lagoon are relatively high except Mn. Chaetomorpha Linium could be used as an excellent indicator for heavy metals accumulation in Korba lagoon.
\end{abstract}

Keywords :Korba lagoon, heavy metals, macro-algae, bio-indicator. 


\section{Résumé}

Les concentrations des métaux lourds ( $\mathrm{Fe}, \mathrm{Zn}, \mathrm{Pb}$ et $\mathrm{Mn}$ ) ont été déterminées dans deux algues vertes (Enteromorpha intestinalis et Chaetomorpha Linium) collectées entre avril 2012 et février 2013 dans neuf stations caractérisées par l'abondance de ces algues durant toute l'année. Le fer a été dosé avec l'absorption atomique à flammeSOLAAR FAAS de type ICE 3300 alorsque les autres éléments ( $\mathrm{Mn}, \mathrm{Pb}$ et $\mathrm{Zn}$ ) ont été dosés avec le four à Graphite. Une évaluation du degré d'eutrophisation a été faite en se basantsur l'étude physico-chimique de 31 échantillons d'eau de surface. Les résultats ont montré que Korba est une lagune eutrophie. La capacité d'accumulation des métaux chez les deux macroalgues varie selon la saison et selon l'espèce. Le potentiel de bioaccumulation des espèces d'algues étudiées sont étroitement liées aux paramètres physicochimiques. L'accumulation des métaux dans l'eausuit l'ordre $\mathrm{Fe}>\mathrm{Zn}>\mathrm{Mn}>\mathrm{Pb}$, tandis que dans Chaetomorpha Linium, Enteromorpha intestinalis et les sédiments suivait le même ordre: $\mathrm{Fe}>\mathrm{Mn}>\mathrm{Zn}>\mathrm{Pb}$. Par comparaison avec des études dans d'autres milieux similaires dans le monde, les niveaux d'accumulation des métaux trouvés dans les algues de la lagune de Korba sont relativement élevés excepté pour le manganèse. Enteromrpha intestinalis et Chaetomorpha Linium sont deux algues largement utilisées comme bioindicateurs de la contamination des écosystèmes. Chaetomorpha linum semble accumule les métaux plus qu'Enteromorpha intestinalis.

Mots-Clés : Lagune de Korba, eutrophisation, éléments traces, macroalgues, bioindicateur

\section{Introduction}

Les zones côtières constituent des espaces complexes et assujettis à une pression démographique, économique et écologique de plus en plus accrue. Ces pressions menacent gravement les ressources naturelles et la viabilité même à long terme des activités économiques. Diverses activités humaines (industrie, agriculture, urbanisation, tourisme...) contribuent à la contamination de l'eau et des sédiments de ces zones et des organismes qui y vivent. Après le rejet dans le milieu aquatique, les polluants organiques et métalliques peuvent rester dans l'eau sous forme dissoute ou être retirés de la colonne d'eau par sédimentation et s'adsorber à la matière organique présente dans le sédiment, (Oueslati.W, 2011; Helali. Med. A,2015). Les métaux lourds ont tendance à être bioaccumulés tout au long des réseaux trophiques. Ils sont absorbés par les algues et bioassimilés par les différentes espèces de la macrofaune benthique (Maanan.M, 2008 ; Rybak et al.,2013 ) et contaminer les prédateurs supérieurs comme les oiseaux aquatiques en concentrant ces métaux présents dans leurs proies (Lucia et al. 2012). 
L’appréciation de la qualité des eaux de surface se base sur la mesure des paramètres physico-chimiques et chimiques ainsi que sur la présence ou l'absence d'organismes et de micro-organismes aquatiques, indicateurs d'une plus ou moins bonne qualité de l'eau. Ces données peuvent être complétées par l'analyse des sédiments.

Pour évaluer la pollution chimique de l'environnement aquatique, nous pouvons utiliser plusieurs matrices qui sont marinés et capables d'accumuler et de conserver la contamination. Depuis ces derniers temps, les algues ont eu un rôle important pour maintenir les milieux aquatiques: en tant que producteurs primaires dans la chaîne alimentaire, une source importante de $\mathrm{O}_{2}$ dans l'eau et de bioindicateur de la pollution. Par conséquent plusieurs algues rouges, brunes et vertes sont utilisées pour examiner l'état de la contamination d'un écosystème donné par certains métaux. Les importants paramètresphysiques et chimiques qui influent la qualité de l'eau sont la température, le $\mathrm{pH}$, la turbidité, l'oxygène dissous (DO), la demande biochimique en oxygène $\left(\mathrm{DBO}_{5}\right)$, les éléments nutritifs (Nitrate-N, Phosphate-P) (Lawson, 2011). Ces paramètres sont des facteurs limitant pour la survie de la flore aquatique.En milieu aquatique, les algues sont connues par leur capacité à fixer et accumuler les éléments naturellement présents dans l'eau, mais aussi les polluants tels que les métaux lourds. Plusieurs études ont montré que la bioaccumulation des métaux lourds par les algues peut être un moyen de contrôle de la pollution et de traitement des eaux usées (Philips, 1997; Chouba et al,2010). La concentration des métaux dans les eaux, les sédiments et les algues de la lagune est fortement liée aux facteurs environnementaux du milieu. Messayaz et Rybak (2013) ont signé que le développement des algues vertes est dépendent des éléments nutritifs ainsi que de $\mathrm{pH}$ du milieu. Rybak et al, 2013 ont montré qu'il y a de nombreux facteurs qui ont un impact sur l'abondance des métaux dans les algues par exemple les processus biologiques des concentrations de métaux dans les sédiments et dans l'eau.

La lagune de Korba est un site Ramasar sur la presqu'île du Cap Bon au Nord de la Tunisie (Fig. 1). Le site d'étude a une superficie moyenne de 369 ha et une longueurde $11 \mathrm{~km}$. Il est séparé de la mer par une barre de sable. Les contributions continentales et maritimes de l'eau dans la lagune sont (inondations et tempêtes) occasionnelles. Malgré sa proximité avec la mer, la lagune de Korba est caractérisée par un climat semi-aride (Bouden et al, 2009). L'écosystème de Korba est une lagune côtière peu profonde (profondeur est inférieure à $1 \mathrm{~m}$ ).La lagune de Korba constitue l'un des meilleurs modèles de biotopes montrant la contamination des lagunes. Notre site d'étude est soumis à diverses pressions associées à une intensification des activités industrielles et agricoles et une augmentation rapide de la population. L'écosystème de Korba, représente un relais pour un grand 
nombre d'oiseaux autour de 3000 oiseaux enregistrés au cours du mois d'Octobre (Hedhili 1998). L'importance écologique de l'environnement de la lagune de Korba nous ramène à évaluer dans un premier temps son état d'eutrophisation, par l'étude des paramètres physico-chimiques durant les deux saisons sèche et humide. Dans un deuxième temps, son état de contamination par l'étude de l'accumulation de métaux dans deux macroalgues Enteromorpha intestinalis et Chaetomorpha Linium. Les résultats ont été discutés et comparés avec d'autres travaux antérieurs.

\section{Materiel et methodes}

Les prélèvements des eaux et des sédiments (31 échantillons) ont été effectués en été 2012 (Fig.1). Seules les eaux ont été prélevées une autre fois en hiver 2013. Les sédiments prélevés par bennes ont été placés dans des sacs plastiques portant la référence puis mis à la congélation $\left(4^{\circ} \mathrm{C}\right)$ pour minimiser les éventuelles réactions chimiques. Les positions de chaque station ont été fixées par un GPS.

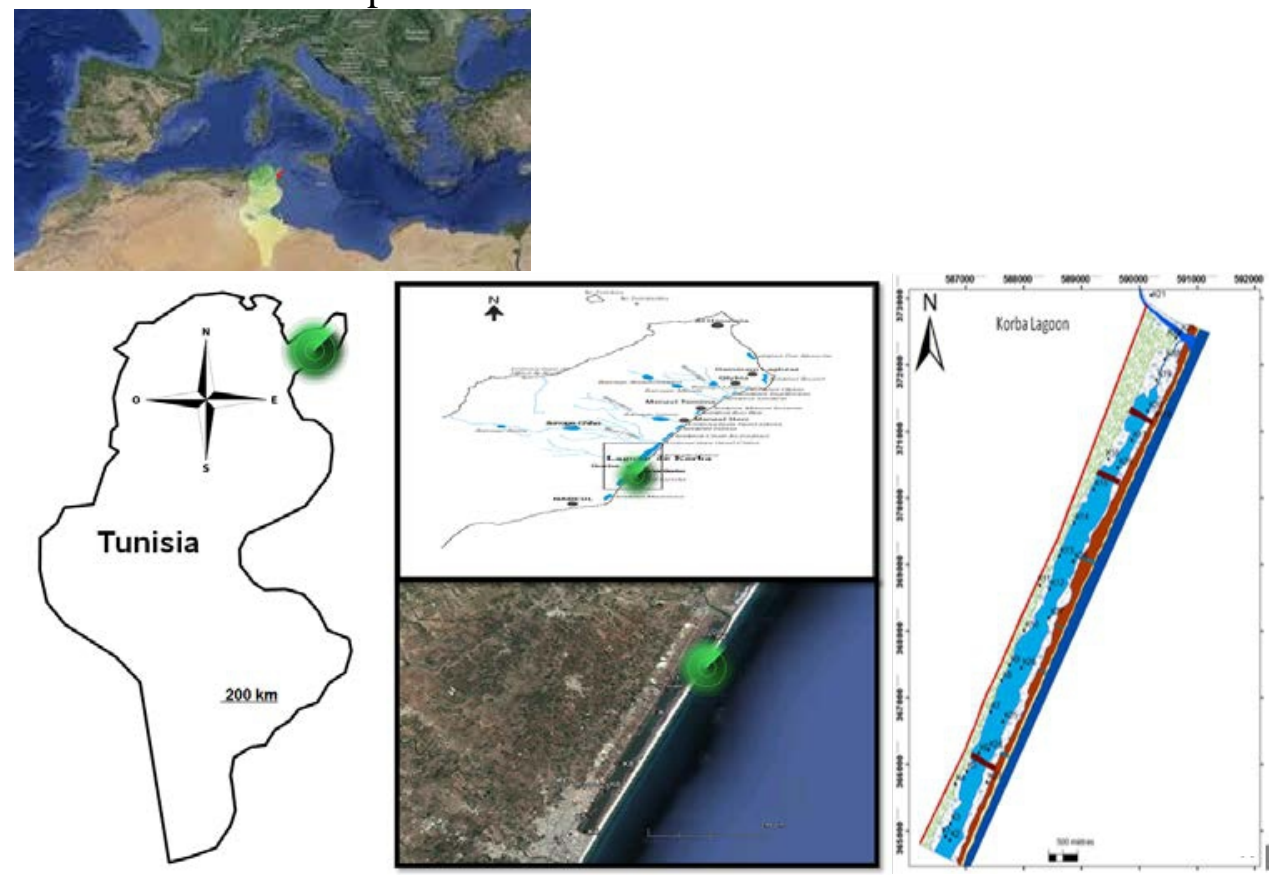

Figure 1. Zone d'étude et emplacement des échantillons

Au laboratoire, chaque échantillon d'eau a été soumis à la dilution nécessaire pour déterminer la concentration des ions majeurs $\left(\mathrm{Ca}^{2+}, \mathrm{K}^{+}, \mathrm{Na}^{+}\right.$ et $\mathrm{Mg}^{+}$) en utilisant la spectrométrie d'absorption atomique. L'alcalinité a été déterminée par titrage avec de l'acide sulfurique M /100. Les chlorures 
sont déterminées par titrimétrie avec du nitrate d'argent $\left(\mathrm{AgNO}_{3}\right)$ (Rodier et al, 2005). Les sulfates ont été déterminés avec le chlorure de baryum $\left(\mathrm{BaCl}_{2}\right.$. $2 \mathrm{H}_{2} \mathrm{O}$ ). La méthode de détermination des sulfates est la précipitation du sulfate de baryum et la détermination de la quantité de précipité par turbidimétrie (Rodier et al, 2005).

Les sels nutritifs ont été analysés par des méthodes colorimétriques automatisées classiques des chaînes Auto-Analyzer. Les concentrations de nitrates ont été déterminées par des analyses colorimétriques après réduction du cadmium en nitrite. Les phosphates ont été analysés par réaction avec du molybdate d'ammonium en milieu acide suivie par une réduction avec de l'acide ascorbique, la concentration est alors déterminée à $885 \mathrm{~nm}$ de longueur d'onde.

Les particules ont été retenues sur les filtres Whatmann GF/F et analysées pour leur teneur en particules en suspension (MS) et en Chlorophylle a (Chl a) conformément à Strickland et Parsons (1972). La demande biologique en oxygène $\left(\mathrm{DBO}_{5}\right)$ a été généralement calculée après 5 jours à $20^{\circ} \mathrm{C}$ et dans l'obscurité.

Les algues recueillies ont été débarrassées de leurs épiphytes rincées sur place par l'eau de la lagune, puis placés dans des sacs en plastique. Au laboratoire, elles sont rincées avec l'eau bidistillée et séchées à $70^{\circ} \mathrm{C}$ pendant $48 \mathrm{~h}$ dans une étuve. La poudre fine obtenue après broyage des algues séchées, a étésoumise à l'action d'un mélange d'acides $\left(\mathrm{HCl}, \mathrm{HNO}_{3}\right.$ et $\mathrm{HClO}_{3}$ ). Les minéralisas sont par la suite filtrés et dilués avec l'eau bidistillée puis stockés dans des flacons à $4^{\circ} \mathrm{C}$ jusqu’à leur analyse (Kaimoussi et al. 2005).

Pour déterminer la concentration en éléments métalliques du sédiment,on a utilisé un spectrophotomètre à flamme SOLAAR FAAS de type ICE 3000. Pour cela nous avons procède à une mise en solution du sédiment à travers une attaque chimique forte (Oueslati, 2011).

Les autres paramètres physicochimiques ont été mesurées in situ à l'aide d'un appareil portatif de type HANNA HI 9829.

\section{Resultats et discussion \\ Principaux paramètres physico-chimiques Température}

La température de l'eau est un facteur écologique qui entraîne d’importantes répercutions écologiques (Rodier et al. 2005). Elle agit sur la densité, la viscosité, la solubilité des gaz dans l'eau, la dissociation des sels dissous, de même que sur les réactions chimiques et biochimiques, le développement et la croissance des organismes vivants dans l'eau.

Dans notre étude, les températures sont comprises entre 21 et $24,5^{\circ} \mathrm{C}$ en hiver et $30-32^{\circ} \mathrm{C}$ en été. Les valeurs sont peu variables dans toutes les 
stations. La répartition spatiale (Fig.2) montre un gradient croissant allant du Sud vers le Nord en hiver et inversement en été. L'augmentation de la température au Sud est probablement due à la faible tranche d'eau et aux eaux de rejet surtout celles des conserveries des tomates qui sont caractérisées par les températures les plus élevées. Les faibles valeurs enregistrées au Nord de la lagune peuvent être expliquéespar l'entrée des eaux froides de la station d'épuration vers la lagune au Nord.

\section{pH}

Le $\mathrm{pH}$ est une mesure de l'acidité de l'eau c'est à dire de la concentration en ions hydrogène $\left(\mathrm{H}^{+}\right)$. Il résume la stabilité de l'équilibre établi entre les différentes formes de l'acide carbonique et il est lié au système tampon développé par les carbonates et les bicarbonates.Les valeurs observées dans notre écosystème varient entre 8 et 9, 25 que ce soit en hiver ou en été. Les cartes de répartition spatiale du pH (Fig.3) montrent que les teneurs les plus faibles sont enregistrées au secteur Nord de la lagune en hiver et ce ci peut être expliqué par l'apport en eau douce de l'oued Chiba. Les valeurs les plus élevées caractérisent la partie Sud et le milieu de la lagune ce qui est probablement dû à l'intrusion des eaux marines au moment des crues. Des augmentations de $\mathrm{pH}$ peuvent se produire suite à des phénomènes d'eutrophisation ou par des rejets d'eaux usées alcalines (Lisec, 2004)

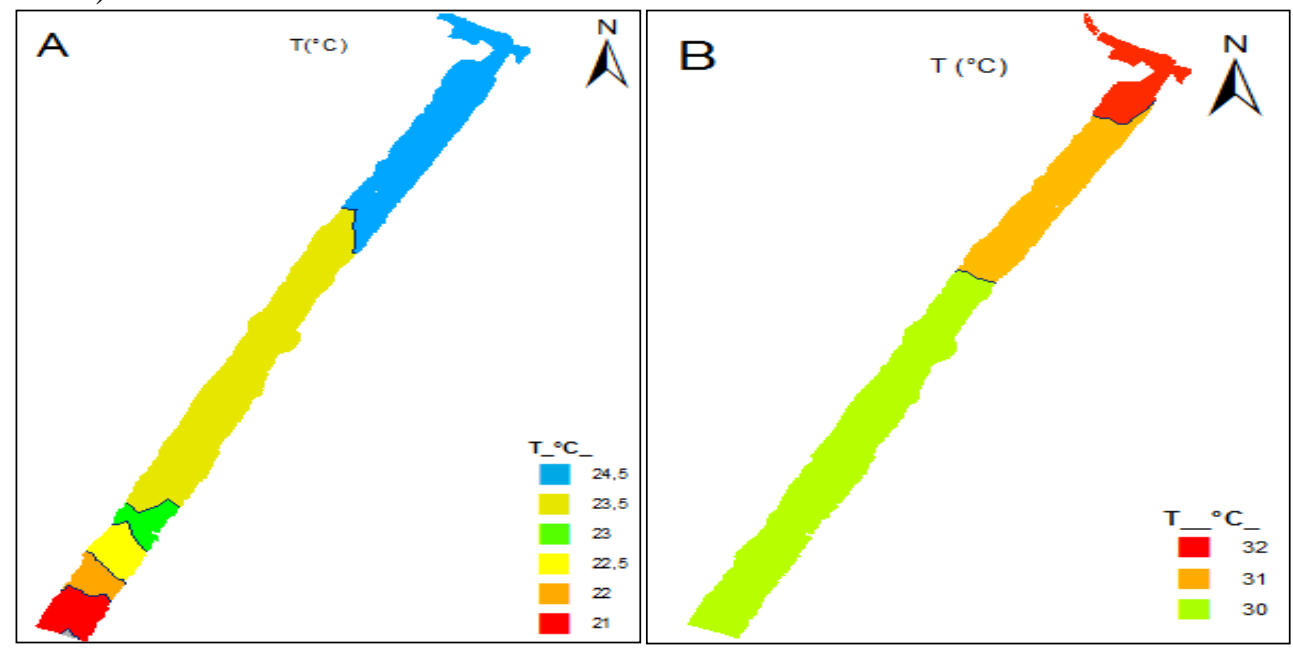

Figure 2. Distribution spatiale des concentrations de la température dans les eaux de surface. A) en hiver B) en été . 


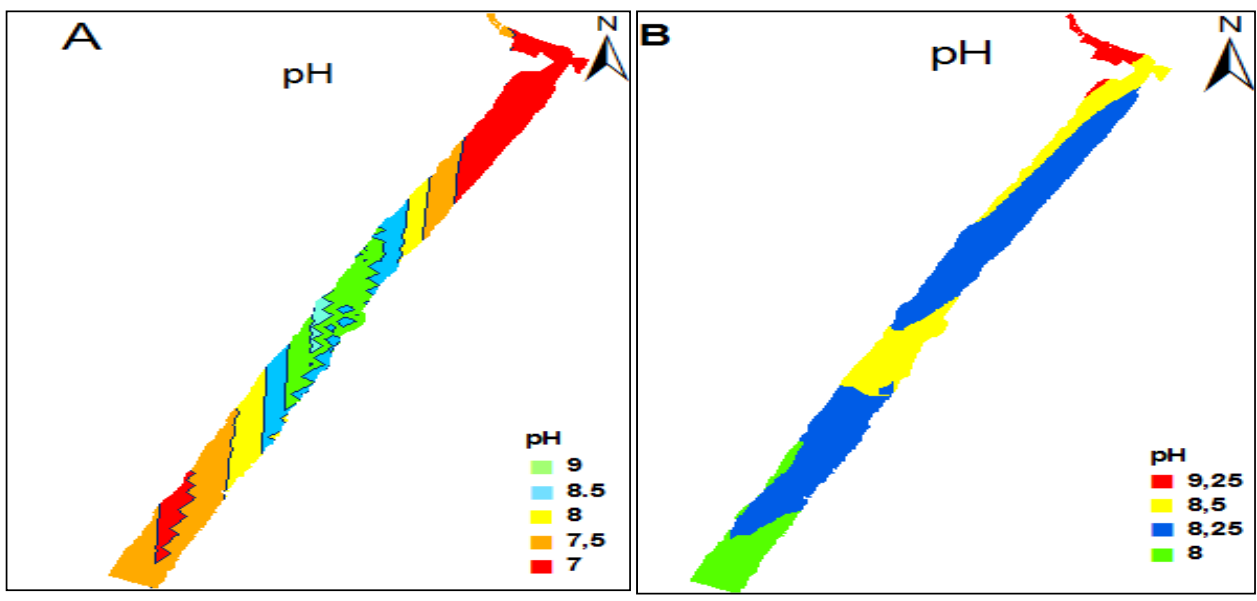

Figure 3. Distribution spatiale des concentrations du $\mathrm{pH}$ dans les eaux de surface. A) en hiver et B) en été .

\section{Salinité}

Les données de la salinité enregistrées indiquent une masse d'eau très saline surtout durant la saison estivale (Fig.4). Entre la partie Sud et la partie Nord de la lagune, la salinité est globalement homogène (38,58 et $78,18 \mathrm{~g} / \mathrm{l}$ respectivement en hiver et en été) avec une chute de salinité pouvant descendre jusqu'à 4,06 g/l au niveau de la station K1. Cette diminution de salinité peut être expliquée par la dilution des eaux de la lagune par les eaux de la Step et les eaux douces de l'Oued Chiba. Les fortes concentrations sont dues au phénomène d'évaporation qui est très intense. Dans les eaux de la lagune, les fortes salinités sont liées d'une part au lessivage des sols salés à alcalis situés entre la dune tyrrhénienne et la côte, et d'autre part, de la dissolution des sels déposés pendant la saison sèche par les eaux de ruissellement.Une prolifération d'algues vertes est remarquée malgré les fortes concentrations de la salinité.

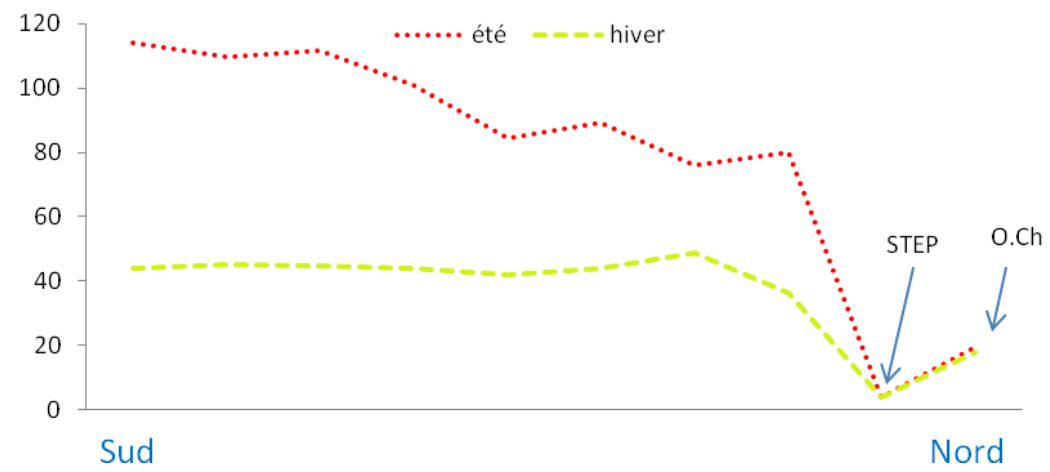

Figure 4: Variation de la salinité des eaux de surface( les flêches montrent les points influencés par la step et l'oued Chiba ) durant les deux saisons. 


\section{Conductivité électrique (EC)}

La conductivité électrique (EC) est une expression numérique de la capacité d'une solution à conduire le courant électrique. La plupart des sels minéraux en solution sont de bons conducteurs. La mesure de la conductivité constitue une bonne appréciation du degré de minéralisation d'une eau où chaque ion agit par sa concentration et sa conductivité spécifique. Les valeurs enregistrées varient entre 7 et $66 \mathrm{mS} / \mathrm{cm}$ en hiver, et entre 7 et 160 $\mathrm{mS} / \mathrm{cm}$ été (Fig. 4). Les valeurs enregistrées en hiver ne montrent pas un écart important, à l'exception de celles qui caractérisent les stations K3, K8 et K7 qui sont influencées par les rejets des eaux usées de faibles conductivités. La répartition des valeurs de la conductivité en juillet montrent un écart très prononcé, en particulier par rapport au niveau de l'extrême sud de la lagune. Les plus faibles valeurs sont enregistrées au niveau des stations influencées par les rejets des usines, du Step et de l'oued Chiba.

Les valeurs les plus élevées marquent le sud et le centre de la lagune au niveau de la passe communiquant avec la mer.

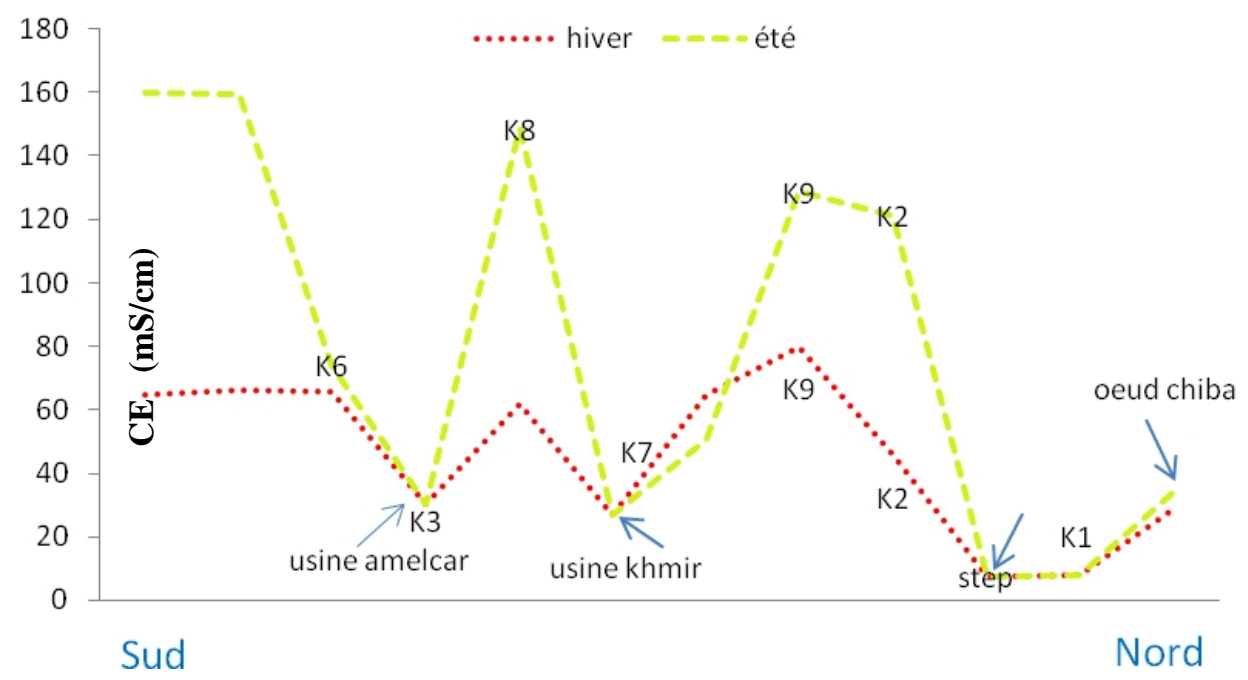

Figure 5: Variation de la conductivité électrique des eaux de surface ( les flêches montrent les points de rejets des usines, du step et de l'oued Chiba) durant les deux saisons.

\section{L'oxygène dissous (OD) et le Potentiel redox (Eh)}

L'oxygène joue le rôle le plus important pour évaluer la qualité biotique des eaux. Il est indispensable à la respiration des organismes et il facilite la dégradation de la matière organique dans les systèmes aqueux. Dans une eau bien oxygénée, les conditions d'oxydation dominent. Quand les concentrations d'oxygène diminuent, le milieu devient plus réducteur ce qui se traduit par une réduction du potentiel redox. 
Dans notre étude, l'évolution saisonnière de l'oxygène dissous montre des concentrations plus élevées durant la période humide que durant la saison sèche. En effet, les teneurs enregistrées (fig.5) varient entre $2 \mathrm{mg} / \mathrm{l}$ et $9 \mathrm{mg} / \mathrm{l}$ et entre $2,5 \mathrm{mg} / \mathrm{l}$ et 9 respectivement en hiver et en été. Durant les deux saisons, les faibles concentrations sont enregistrées dans la partie sud. L'insuffisance d'oxygène dissous pourrait expliquer les faibles valeurs du potentiel redox (fig.6) dont la valeur moyenne est de 79,37 $\mathrm{mV}$ en été et de109,89 mV en hiver. Celui-ci est associé aux réactions d’oxydoréduction et à la biodégradation des composés organiques. Lorsque l'oxygène est trop faible, la décomposition de la matière organique soluble devient très lente et le potentiel redox devient faible montrant ainsi que le milieu est réducteur. Par ailleurs, la décomposition des algues mortes induit également une consommation d'oxygène. Lorsque l'eau est trop peu oxygénée on parlera donc des conditions d'anaérobie.

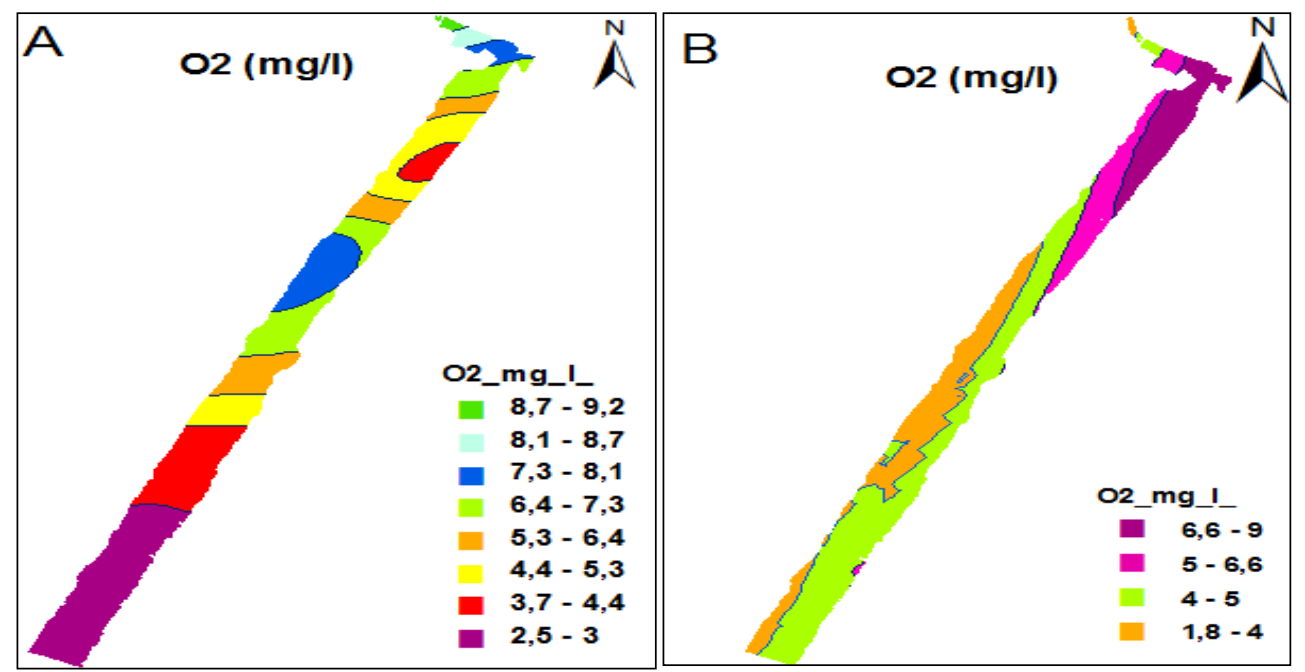

Figure 6. Distribution spatiale des concentrations d' $\mathrm{O}_{2}$ dans les eaux de surface. A) en hiver et B) en été.

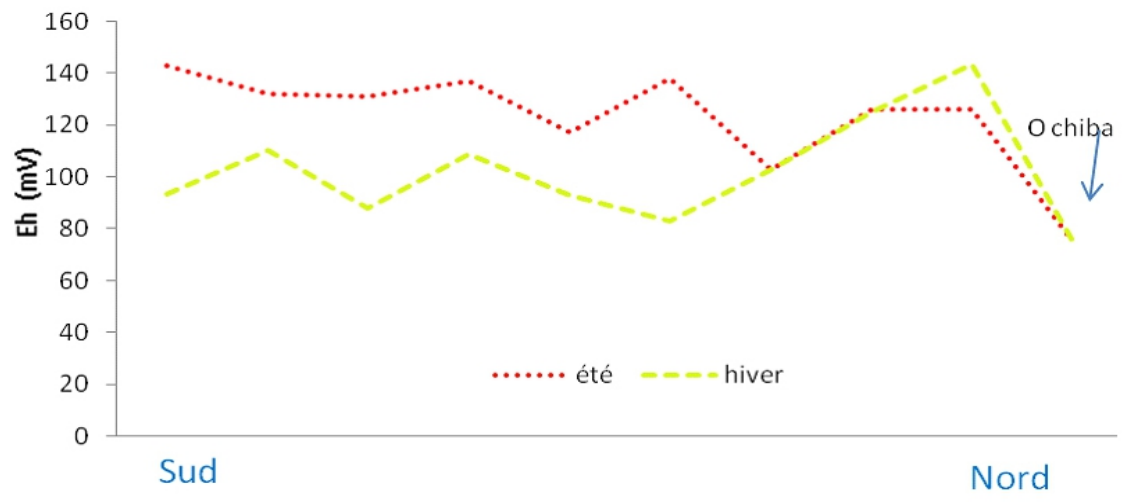

Figure 7: Variation du potentiel redox (Eh) des eaux de surface durant les deux saisons. 


\section{Matières en suspension (MES)}

Les matières en suspension comprennent toutes les matières minérales ou organiques qui ne se solubilisent pas dans l'eau. Elles incluent les argiles, les sables, les limons, les matières organiques et minérales de faible dimension, le plancton et autres micro-organismes de l'eau. La quantité de matière en suspension varie notamment selon les saisons et le régime d'écoulement des eaux. Pour les deux saisons humide et sèche, les valeurs des MES enregistrées sont élevées. La répartition spatiale (Fig. 8) montre que les charges de MES en période estivale sont supérieures (1370mg/l) à celles mesurées en période hivernale (crue) (200mg/l). L'augmentation des MES entraîne une faible transparence réduisant la pénétration de la lumière dans l'eau modifiant la turbidité des eaux ainsi la réduction de la photosynthèse (Philippe, 1994).

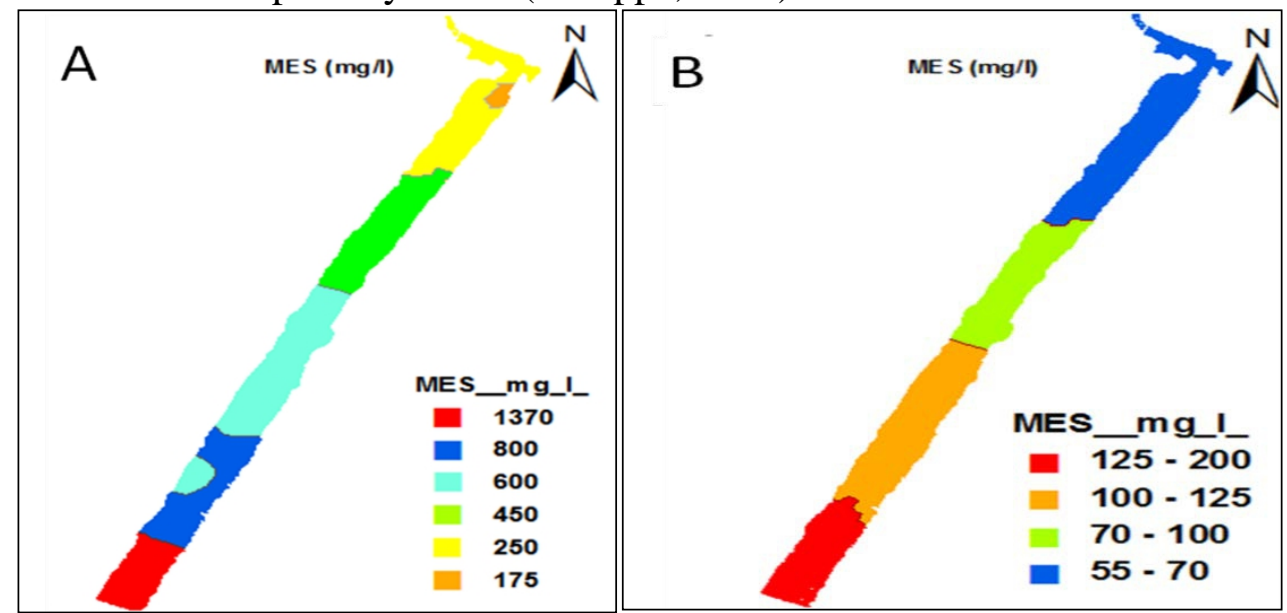

Figure 8. Distribution spatiale des concentrations de MES dans les eaux de surface. A) en étéet B) en hiver.

\section{Chlorophylle a (Ch a)}

Le contenu en chlorophylle constitue une mesure sensible de la quantité d'organismes photosynthétiques et d'algues et, par conséquent, du degré d'eutrophisation de l'eau.

Les teneurs en chlorophylle a varient entre 75 et $300 \mu \mathrm{g} / \mathrm{l}$ en été et entre 124 et $248 \mu \mathrm{g} / \mathrm{l}$ en hiver, ce qui est considéré comme des valeurs relativement élevées. Les plus faibles teneurs sont enregistrées au Nord du secteur d'étude près de la station d'épuration. Les plus fortes teneurs caractérisent les eaux prélevées au Sud et au centre. Nous avons noté que la répartition spatiale du chlorophylle a est similaire durant les deux saisons. Les valeurs élevées indiquent que le milieu est hypereutrophe. 


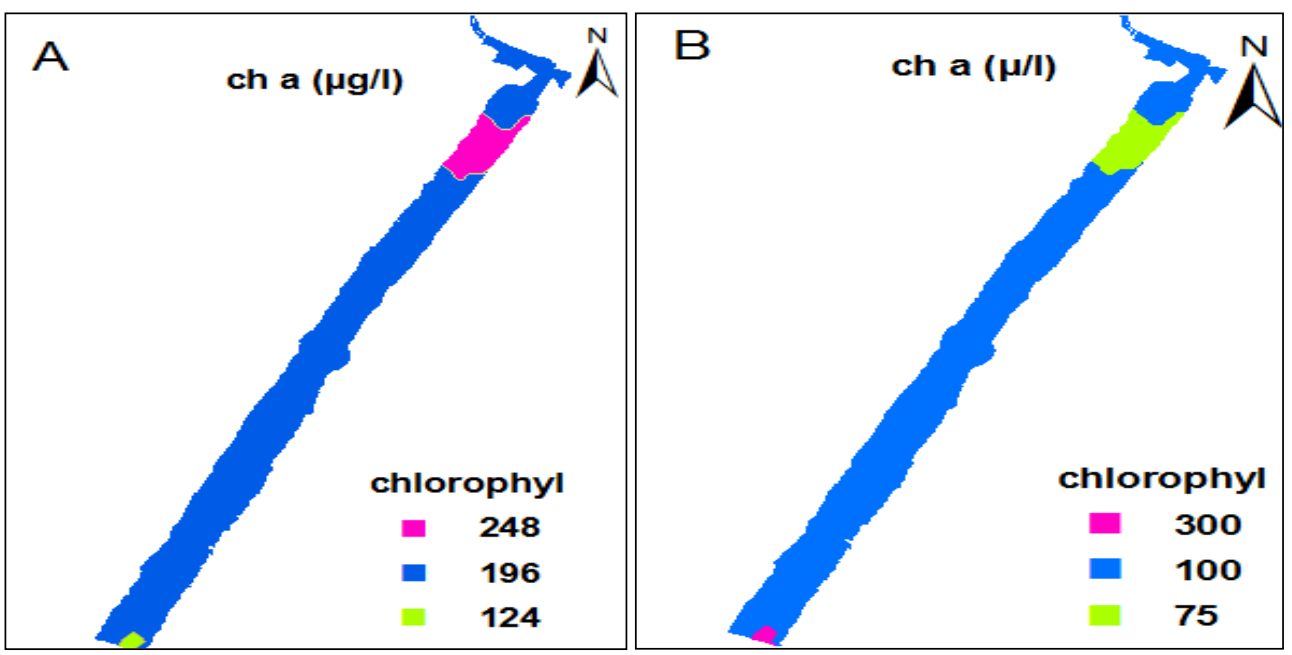

Figure 9.Distribution spatiale des concentrations de la chlorphylle a dans les eaux de surface. A) en hiver B) en été .

\section{$\mathrm{DBO}_{5}$ (Demande Biochimique en Oxygène pendant cinq jours)}

Les valeurs enregistrées sont similaires dans les deux saisons. Les concentrations oscillent entre 48 et $312 \mathrm{mg} \mathrm{O}_{2} /$ l. Les ordres de grandeur de la $\mathrm{DBO}_{5}$ enregistrés dans cette étude sont largement supérieursaux normes (Fig.10). De tel résultat pourrait être expliqué par les apports de composés organiques biodégradables ou non et par les effluents domestiques et industriels dans la lagune sans traitement préalable. Les teneurs en phytoplancton et en chlorophylle a sont d'autres importantes sources de la demande en oxygène (Heiskary et Markus, 2001). Ces fortes teneurs témoignent d'une grande charge en matière organique.

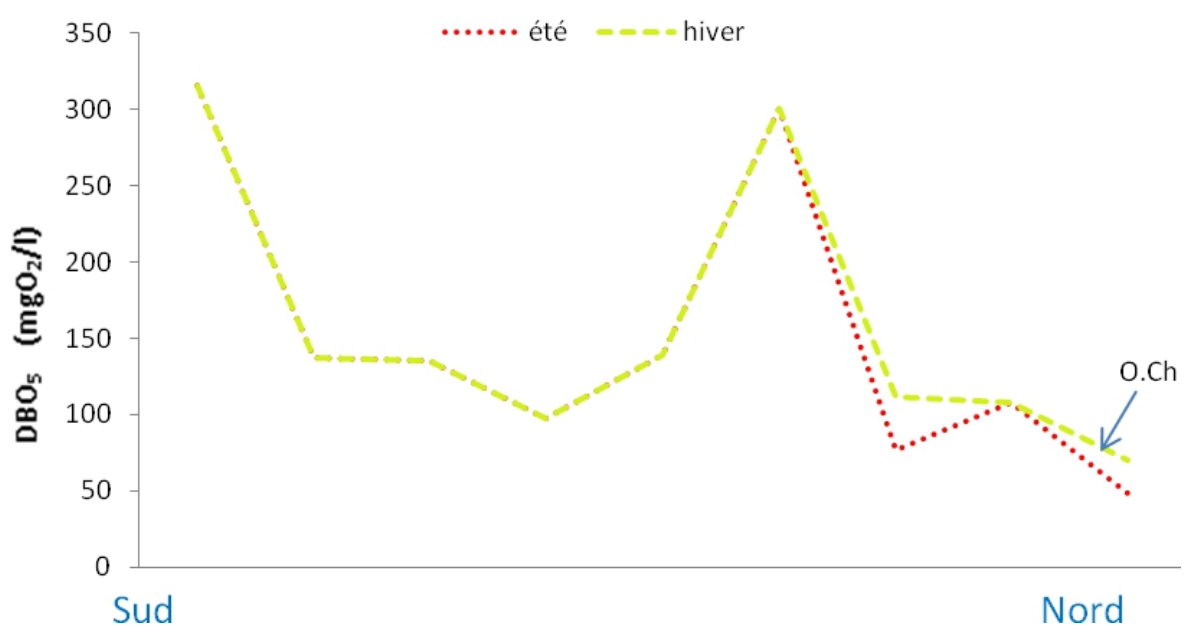

Figure 10: Variation de $\mathrm{DBO}_{5}$ des eaux de surface durant les deux saisons.

Substances eutrophisantes : 


\section{les nitrates}

Dans les eaux de la lagune de Korba, la concentration en nitrate varie entre 2,9 mg/l et 18,5 mg/l en hiver et entre $0,9 \mathrm{mg} / \mathrm{l}$ et $18,8 \mathrm{mg} / \mathrm{l}$ en été. Les teneurs les plus fortes en nitrates sont enregistrées au Sud c'est-àdire dans les eaux les moins oxygénées et les faibles concentrations sont enregistrées au niveau des stations proches du Step et de l'Oued Chiba. Les nitrates présents dans l'eau peuvent provenir des sources indirectes à savoir la décomposition des algues mortes qui induit également une consommation de l'oxygène. Lorsque l'eau est trop peu oxygénée, les conditions d'anaérobiose risquent également de se traduire par une accumulation de composés ammoniaqués et de nitrates. Les valeurs obtenues dans notre site sont probablement dues aux multiples apports d'effluents arrivant à cette lagune. En effet, les effluents industriels, agricoles et les déjections humaines élèvent les teneurs en nitrates des eaux et sont témoins de la dégradation de la qualité de l'eau.

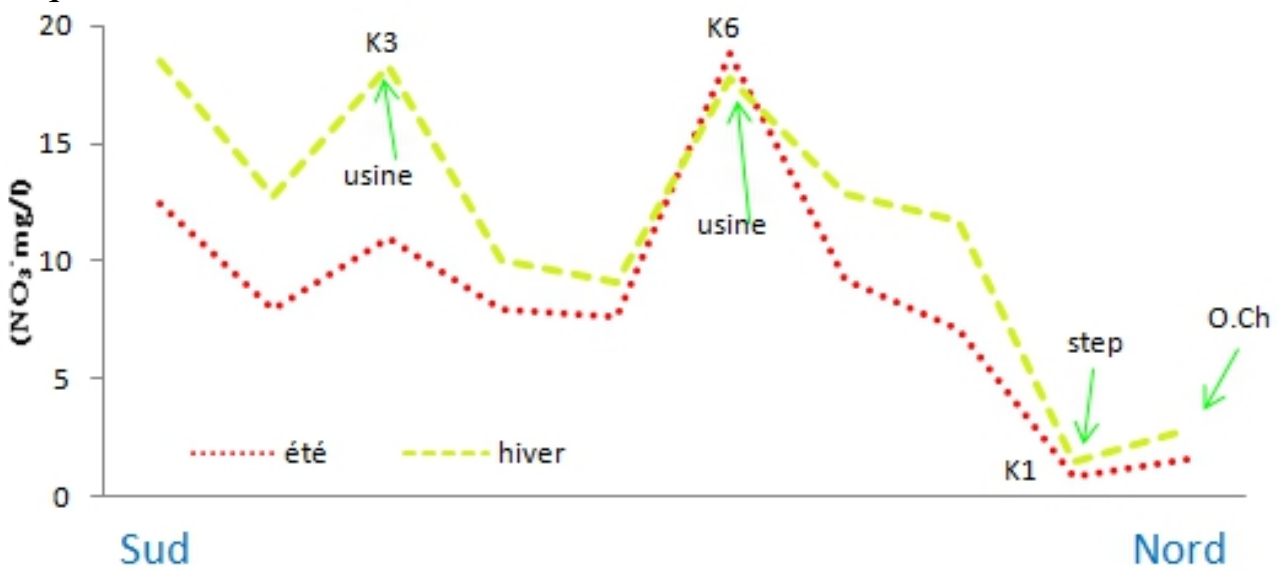

Figure 11: Variation des nitratesdans des eaux de surface durant les deux saisons.

\section{Les orthophosphates}

Les concentrations moyennes des orthophosphates varient entre 57,11 et 66,58 mg/l respectivement en hiver et en été. Les apports en phosphore dans un milieu aquatique récepteur, ont une origine diffuse (érosion des sols, lessivage des engrais des zones cultivées) et une origine ponctuelle (effluents urbains, effluents industriels).Les valeurs élevées en sels nutritifs (nitrates et phosphates) sont responsable en premier lieu de la prolifération des macroalgues sur toutes la surface du complexe lagunaire de Korba. L'eutrophisation peut déjà se manifester à des concentrations relativement basses en phosphates (50 $\mu \mathrm{g} / \mathrm{l}$ ) (Rapin A and all, 1989). On considère que la situation est très critique à partir d'une concentration de plus de $0,1 \mathrm{mg} \mathrm{NO}_{3}{ }^{-}$ /l (Chambers PA and al, 2001). Globalement, pour l'ensemble des stations 
suivies, les charges en composés azotés et en $\mathrm{PO}_{4}{ }^{3-}$ ont été très élevées et traduisent une très forte eutrophisation des eaux de la lagune.

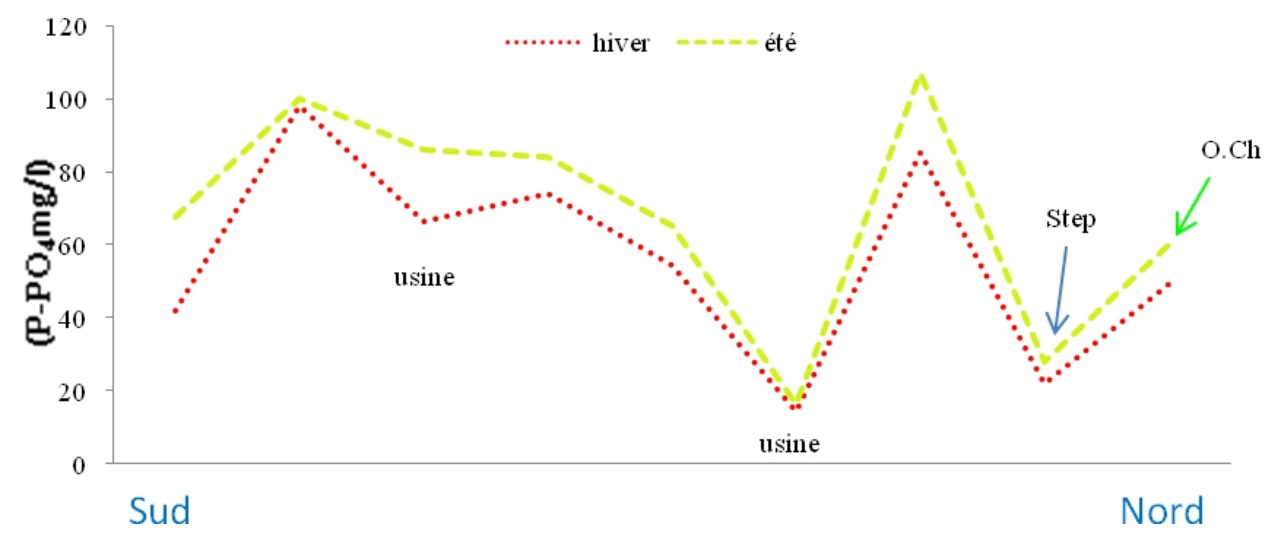

Figure 12: Variation des orthophosphates dans des eaux de surface durant les deux saisons.

\section{Le faciès géochimique des eaux de la lagune de Korba:}

La projection de la composition en éléments majeurs, exprimée en mg/l des différents échantillons d'eau de surface de la lagune de Korba et durant les deux saisons hivernale et estivale a été réalisée dans le diagramme de Piper à l'aide d'un logiciel d'hydrochimie « DIAGRAMMES ». Le but est de déterminer le faciès géochimique de ces eaux.

Une eau sulfatée chlorurée sodique en hiver et une eau sulfatée sodique en été (Fig.13). Les trois éléments sulfates, sodium et chlore sont les ions dominants dans les eaux de la lagune. $\mathrm{Na}^{+}$et $\mathrm{Cl}^{-}$peuvent avoir la même origine comme la dissolution de la halite et aussi une origine marine au moment des crues car le faciès chloruré-sodique est faciès marin. Ces éléments contrôlent la charge saline de ces eaux. Alors que les sulfates enrichis dans les eaux peuvent être expliqués par la disponibilité du sulfure d'hydrogène lors de la dégradation des algues mortes. Briand (1989) a bien montré que les protéines des algues se composent de 2 à $5 \%$ de soufre de leurs poids sec. Le soufre présent dans l'eau de la lagune est indispensable pour la prolifération des algues.L'algue même peut être la source du soufre.

L’état de putréfaction avancée des algues est facilement identifiable visuellement dans la lagune de Korba. En effet, exposée au soleil, la surface des amas d'algues sèches forment une croûte blanchâtre. Sous cette croûte

règne une forte obscurité interrompant la photosynthèse et créant des conditions de fermentation qui évoluent de l'aérobie vers l'anaérobie. Nous avons ressenti l'œuf pourri, ce qui peut être expliqué par la libération du sulfure de l'hydrogène lors de la décomposition des algues. Les microorganismes sulfato-réducteurs, très abondants dans l'eau, utilisent les sulfates présents dans les protéines des algues comme source d’oxygène, ce qui entraîne la formation du sulfure d'hydrogène $\left(\mathrm{H}_{2} \mathrm{~S}\right)$. 
A

Diagramme de Piper

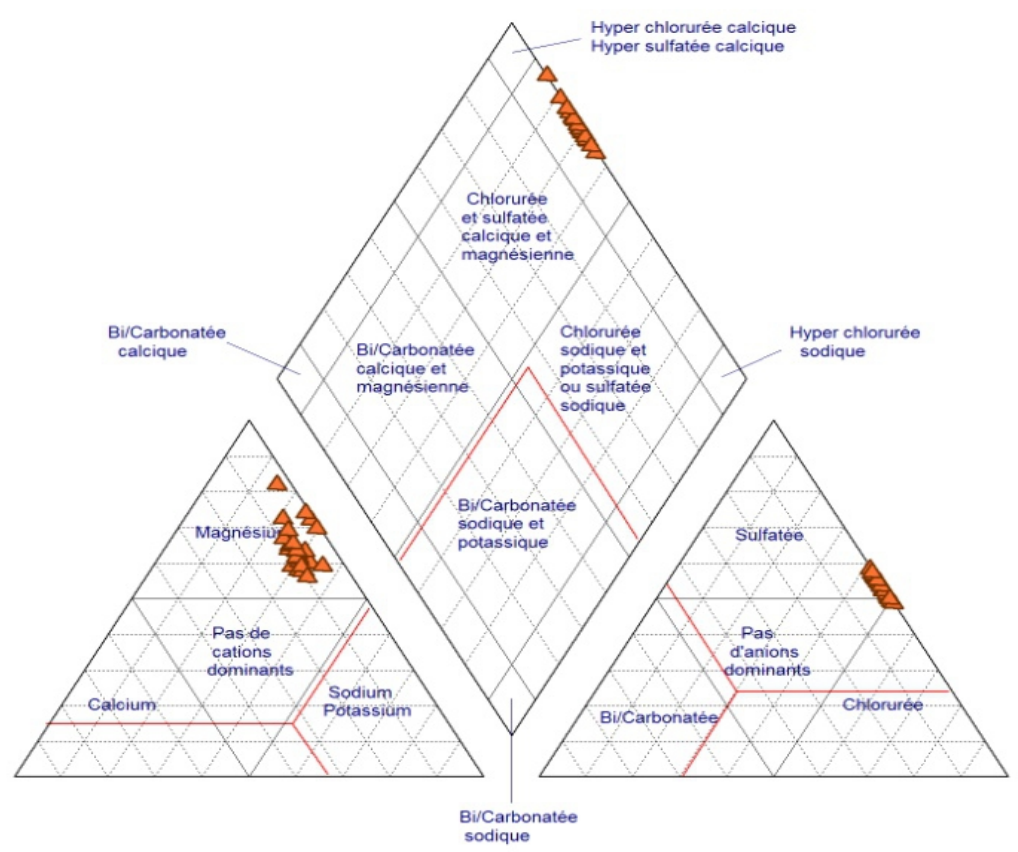

B

Diagramme de Piper

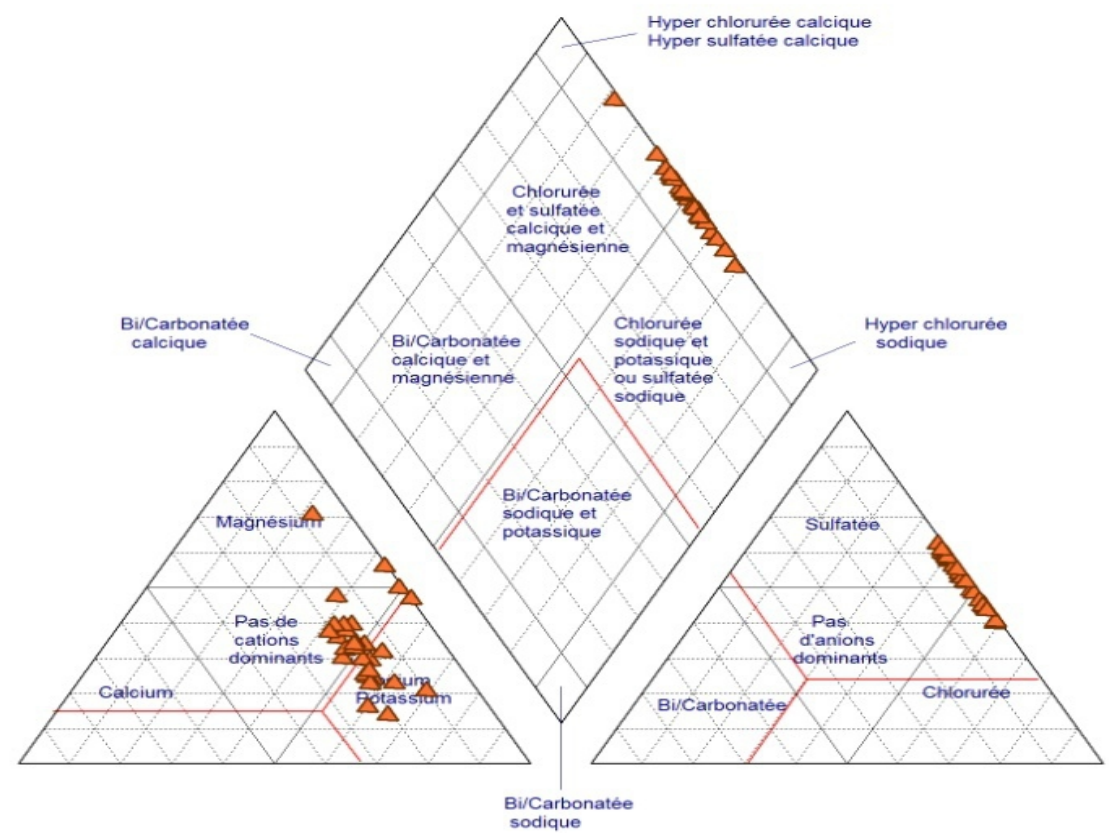

Figure 13: Diagramme de Piper des eaux de surface de la lagune de Korba : en été (A) et en hiver (B). 


\section{Métaux lourds dans les eaux et les sédiments de la lagune}

Pour se nourrir, l'algue fabrique des sucres à partir de l'eau et du gaz carbonique dansl'eau. Les algues produisent plus la moitié de l'oxygène de notre atmosphère, directement en absorbant les sels minéraux contenus dans l'eau. Donc l'eau circule dans les thalles et les algues concentrent les minéraux et les métaux. La biodisponibilité des métaux dans l'eau est fortement liée à la présence de ces derniers dans les sédiments. Le sédiment représente le puits d'accumulation des métaux lourds mais aussi la source. De ce fait, pour bien comprendre la bioaccumulation des métaux dans la flore aquatique d'un tel écosystème, il est indispensable de savoir la tendance d'accumulation des métaux étudiés dans l'eau et le sédiment avant de l'étudier dans les algues même.D'après les tableaux 3 et 4 nous constatons que les sédiments de surface de la lagune de Korba ont tendance d'accumuler les métaux dans l'ordre : $\mathrm{Fe}>\mathrm{Mn}>\mathrm{Zn}>\mathrm{Pb}$. Ceci est confirmé par le travail de Bouden, 2006 dans la même lagune. L’eau a tendance à accumuler le fer, le zinc, le manganèse puis le plomb : $\mathrm{Fe}>\mathrm{Zn}>\mathrm{Mn}>\mathrm{Pb}$.

Tableau 3 : Concentrations de $\mathrm{Pb}, \mathrm{Zn}, \mathrm{Mn}$ et Fe dans l'eau ( en ppb) des sites étudiés

\begin{tabular}{|c|c|c|c|c|}
\hline & Pb & Zn & Mn & Fe \\
\hline K1 & 403,61 & 543,56 & 347,35 & 20638,15 \\
\hline K2 & 85,66 & 814,18 & 492,35 & 32494,95 \\
\hline K3 & 23,40 & 854,71 & 241,95 & 35418,65 \\
\hline K4 & 26,36 & 873,03 & 205,6 & 40720,7 \\
\hline K5 & 13,13 & 753,05 & 535,55 & 22585 \\
\hline K6 & 24,67 & 877,75 & 132,65 & 39664,45 \\
\hline K7 & 50,80 & 914,52 & 173,1 & 44401,85 \\
\hline K10 & 74,20 & 965,66 & 132,7 & 49885,2 \\
\hline K11 & 54,21 & 916,14 & 472,1 & 20638,15 \\
\hline K12 & 98,54 & 854,21 & 481,3 & 32494,95 \\
\hline K13 & 154,54 & 890,9 & 187,89 & 35418,65 \\
\hline K14 & 95,5 & 683,12 & 204,9 & 40720,7 \\
\hline K15 & 201 & 412,65 & 254,51 & 22585 \\
\hline K16 & 241 & 365,41 & 189,57 & 39664,45 \\
\hline K17 & 254,65 & 412,6 & 301,46 & 44401,85 \\
\hline K18 & 154,35 & 541,48 & 211,31 & 49885,2 \\
\hline K19 & 163,47 & 647,31 & 412,6 & 20638,15 \\
\hline K20 & 284,3 & 456 & 384,54 & 32494,95 \\
\hline K21 & 89,65 & 542,17 & 278,65 & 35418,65 \\
\hline K24 & 84,6 & 203,16 & 99,53 & 40720,7 \\
\hline K25 & 188,4 & 466,6 & 200,4 & 22585 \\
\hline K26 & 79,5 & 193,8 & 271,31 & 39664,45 \\
\hline K27 & 247,35 & 641,48 & 189,65 & 44401,85 \\
\hline K28 & 36,87 & 270,35 & 175,64 & 49885,2 \\
\hline K30 & 38,41 & 600,27 & 354,14 & 40720,7 \\
\hline K31 & 124,5 & 249,55 & 187,54 & 22585 \\
\hline & & & & \\
\hline
\end{tabular}


Tableau 4 : Concentrations de $\mathrm{Pb}$, Zn, Mn et Fe dans le sédiment (ppm) des sites étudiés

\begin{tabular}{|c|c|c|c|c|}
\hline & $\mathrm{Pb}$ & $\mathrm{Mn}$ & $\mathrm{Zn}$ & $\mathrm{Fe}$ \\
\hline K1 & 134,67 & 361,51 & 244,29 & 395,37 \\
\hline K2 & 43,39 & 341,94 & 269,08 & 319,92 \\
\hline K3 & 8,29 & 351,91 & 60,38 & 848,93 \\
\hline K4 & 0,20 & 231,90 & 204,94 & 3825,77 \\
\hline K5 & 221,70 & 426,13 & 166,83 & 3432,00 \\
\hline K6 & 47,21 & 269,97 & 222,16 & 3755,73 \\
\hline K7 & 236,57 & 230,23 & 64,60 & 17477,02 \\
\hline K8 & 85,58 & 350,62 & 171,39 & 1810,79 \\
\hline K9 & 229,39 & 326,93 & 148,92 & 1800,59 \\
\hline K10 & 21,67 & 515,60 & 48,96 & 3902,39 \\
\hline K11 & 184,10 & 514,34 & 122,66 & 4265,28 \\
\hline K12 & 67,91 & 466,78 & 241,12 & 4376,96 \\
\hline K13 & 196,10 & 490,90 & 58,57 & 8402,46 \\
\hline K14 & 53,87 & 283,42 & 77,83 & 2929,03 \\
\hline K15 & 34,97 & 329,82 & 191,10 & 5298,80 \\
\hline K16 & 104,64 & 285,46 & 94,00 & 2277,41 \\
\hline K17 & 124,83 & 405,25 & 193,89 & 4070,54 \\
\hline K18 & 142,86 & 534,38 & 91,64 & 9542,49 \\
\hline K19 & 97,11 & 566,71 & 45,08 & 12533,48 \\
\hline K20 & 211,20 & 629,47 & 542,17 & 10023,49 \\
\hline K21 & 77,40 & 575,38 & 203,16 & 783,33 \\
\hline K22 & 96,77 & 278,32 & 232,52 & 572,55 \\
\hline K23 & 1,92 & 343,20 & 192,32 & 566,44 \\
\hline K24 & 78,32 & 171,72 & 46,60 & 173,13 \\
\hline K25 & 188,55 & 219,23 & 93,80 & 30,43 \\
\hline K26 & 85,63 & 256,18 & 64,49 & 469,46 \\
\hline K27 & 238,71 & 236,48 & 70,35 & 316,77 \\
\hline K28 & 20,98 & 244,40 & 400,28 & 783,78 \\
\hline K29 & 165,55 & 233,82 & 380,57 & 1185,43 \\
\hline K30 & 21,56 & 368,10 & 249,54 & 996,02 \\
\hline K31 & 179,96 & 174,20 & 59,18 & 263,84 \\
\hline
\end{tabular}

\section{Analyse statistique}

Afin de visualiser la relation et l'interaction des paramètres physicochimiques et les métaux lourds entre eux et la qualité des eaux de la lagune, nous avons réalisé à l'aide du logiciel STATISTICA 12, une Analyse en Composantes Principales (ACP) en utilisant les mêmes séries de données mensuelles des 17 variables retenues. L'ACP a été effectuée sur des données centrées et réduites par rapport aux moyennes et écart-types de ces variables. 

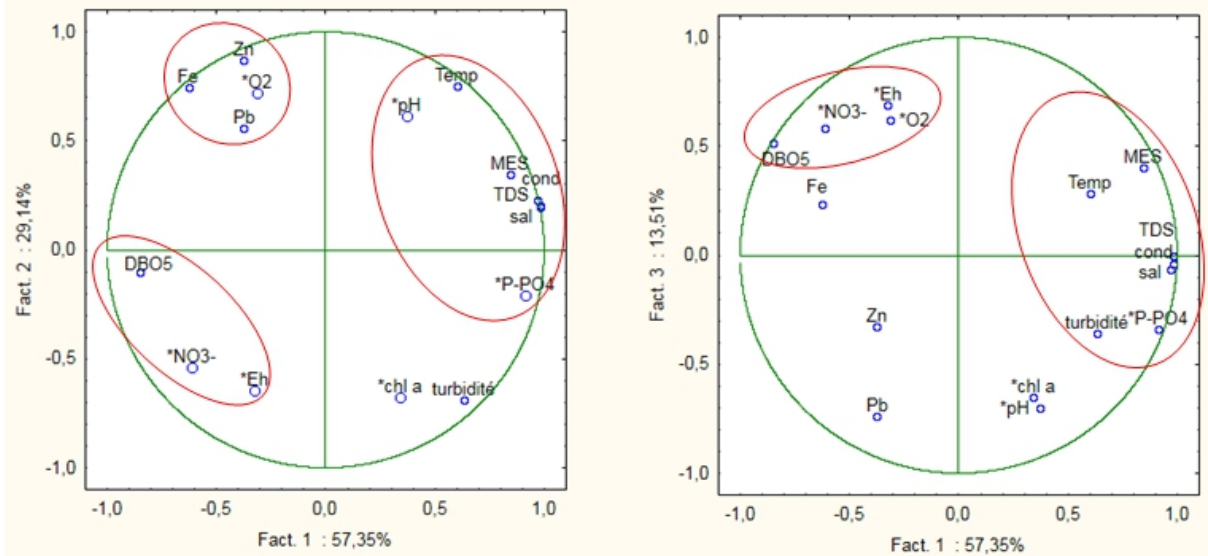

Figure 14: Approche graphique de l'ACP des paramètres physico-chimiques et des métaux dans l'eau selon les plans F1xF2 et F1xF3

Les résultats nous ont permis de retenir trois axes F1,F2 et F3. Les projections faites sur les trois axes montrent que : L'axe 1 résume 57,35\% de l'information et décrit les variables liées à la minéralisation $\left(\mathrm{P}_{-} \mathrm{PO}_{4}\right.$, conductivité, salinité,TDS,température, MES etturbidité). Il peut être considéré comme un gradient de minéralisation lié à l'intensité de la pollution apportée par les rejets d'eaux usées (Fig.14).

L'axe F2 résume 29,14\% de l'information et il est représentatif des métaux. Donc de l'activité anthropique (Fig. 14).

L'axe F3 résume 13,51\% de l'information et montre une corrélation positive entre le nitrate, l'oxygène dissous,le $\mathrm{DBO}_{5}$ et le potentiel d'oxydoréduction Eh. Il est principalement lié à l'activité biologique dans l'eau. Ceci pourrait s'expliquer par le processus de la photosynthèse réalisé par le phytoplancton. L'axe F3 peut être considéré comme un gradient d'eutrophisation (Fig. 14).

\section{les algues indicatrices de la contamination de la lagune}

Dans le but de démontrer la capacité de la bioaccumulation métallique des macroalgues et leur pouvoir de nous informer sur la qualité chimique des eaux de la lagune, on s'est intéressé à la détermination de la gamme de concentration en métaux traces (Plomb, Zinc, Fer et Manganèse) dans deux macroalgues vertes (Enteromorpha intestinalis, Chaetomorpha linum) dans la lagune de Korba. Descollectes saisonnières ont été faites dans la lagune durant la période avril 2012 et février 2013 en raison de l'abondance de ces espèces durant toute la période de récolte. Les variations saisonnières des concentrations des métaux $(\mathrm{Pb}, \mathrm{Zn}$, Fe et $\mathrm{Mn})$ chez les deux espèces d'algues sont présentées dans les figures 15, 16 et 17. 
-Chaetomorpha linium : Les variations des concentrations de Mn sont presque constantes (Fig.15-A). Ces concentrations ont atteint respectivement des valeurs de 56,93 $\mu \mathrm{g} / \mathrm{g}$ MS au printemps et de 57,12 $\mu \mathrm{g} / \mathrm{g}$ MS et de 59,05 $\mu \mathrm{g} / \mathrm{g} \mathrm{MS}$ en hiver et ont chuté pendant l'automne où elles marquent la plus faible concentration $(18,5 \mu \mathrm{g} / \mathrm{g} \mathrm{MS})$. La teneur en $\mathrm{Zn}$ est révélée constante pendant les deux saisons(été et hiver) avec un maximum de $87,91 \mu \mathrm{g} / \mathrm{g} \mathrm{MS}$ en automne et un minimum de $49,95 \mu \mathrm{g} / \mathrm{g}$ MS est atteint en printemps. Pour le $\mathrm{Pb}$, une teneur de $14,3 \mu \mathrm{g} / \mathrm{g} \mathrm{MS}$ est notée en période printanière et continue à augmenter en été pour atteindre un maximum de 17,29 $\mu \mathrm{g} / \mathrm{g} \mathrm{MS}$. Une diminution brusque pendant l'automne est enregistrée avec un minima 4,7 $\mu \mathrm{g} / \mathrm{g}$ MS (Fig. 15-A). Pour le fer, des teneurs élevées ont été enregistrées au printemps et en été avec un maximum printanier de $762,78 \mu \mathrm{g} / \mathrm{g}$ MS et un minimum en automne de $234 \mu \mathrm{g} / \mathrm{g}$ MS(Figure $15-\mathrm{B})$.

-Enteromorpha intestinalis : la teneur maximale en Mn est révélée en été $(37,53 \mu \mathrm{g} / \mathrm{g} \mathrm{MS})$. Les concentrations ne varient pas beaucoup durant le reste de l'année (Fig. 16-A).La teneur en Zn a atteint les valeurs les plus élevées en hiver et en automne avec un maximum automnal de 94,86 $\mathrm{g} / \mathrm{g}$ MS et reste constante pendant le printemps et l'été avec un minimum de $41,37 \mu \mathrm{g} / \mathrm{g} \mathrm{MS}$. La teneur en $\mathrm{Pb}$ est faible pendant toutes les saisons variant

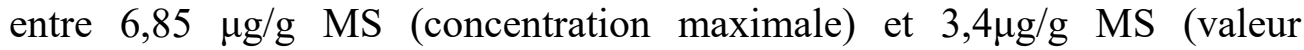
minimale) respectivement en hiver et auprintemps (Fig.16-A).Les concentrations en fer sont élevées au printemps et en hiver avec un maximum hivernal de $826 \mu \mathrm{g} / \mathrm{g} \mathrm{MS}$. Cette augmentation chute en automne à la plus faible concentration $(266 \mu \mathrm{g} / \mathrm{g}$ MS) (Fig.16-B).

Les fluctuations saisonnières des teneurs en métaux dans les deux macroalgues ont montré des variations significatives selon l'espèce et la saison de prélèvement. Les résultats ont montré que les teneurs en différents métaux analysés ont diminué en automne excepté le Zn. Ceci s'explique par l'élévation de la température durant cette période. Phillips,1994 a révélé que les niveaux d'accumulation des métaux dans les macrophytes aquatiques sont généralement faibles dans les mois les plus chauds de l'année en raison des taux de croissance élevés.La variation saisonnière de l'accumulation du métalestexpliquée par la disponibilité du métal dans l'eau (Phillips, 1994), par le temps de submersion de l'algue etpar les paramètres hydrologiques de l'eau (Akcali et Kucuksezgin, 2011).Ces fluctuations saisonnières sont dépendantes de l'âge de l'algue même.En effet la biodisponibilité des métaux est liée à l'organisme considéré et à son stade de développement. Barnett et Ashcroft, 1985 ont enregistré une augmentation de la teneur en Fe, Mn, Zn et $\mathrm{Pb}$ dans les thalles principaux par comparaison à ceux en développement.

La Chaetomorpha Linium a montré un pouvoir accumulateur des métaux étudiés plus important que Enteromorpha intestinalis (Fig.17). Cette étude montre que les deux espèces étudiées ontune préférence 
d'accumulation comme suitFe $>\mathrm{Zn}>\mathrm{Mn}>\mathrm{Pb}$.Cette affinité est en concordance avec celle trouvée dans d'autres travaux montrant que les macroalgues des régions tempérées accumulent beaucoup de $\mathrm{Fe}, \mathrm{Zn}, \mathrm{Mn}$ et $\mathrm{Pb}$ (Müller et al., 1993;Kaimoussi et al., 2005;Chouba et al.,2010).

Afin d'évaluer le niveau de contamination de la lagune de Korba, nous avons comparé les résultats de notre étude avec d'autres travaux sur les algues vertes dans des environnements contaminés et non contaminés.

Il a été rapporté que les résidus de zinc dans Chaetomorpha Linium prélevée dans les eaux polluées varient dans la gamme 50 - $150 \mu \mathrm{g} / \mathrm{g}$ MS (Say et al.,1990;Brown et al., 1999 et Sawidis et al, 2001). Villares et al.,(2002) ont considéré qu'une teneur en Zn de $29 \mu \mathrm{g} / \mathrm{g}$ MS dans Enteromorpha intestinalis caractérise les sites contaminés. Chouba et al en 2010 ont déclaréle lac Nord de Tunis un site pollué en Zinc avec une moyenne de $44 \mu \mathrm{g} / \mathrm{g}$ MS dans Chaetomorpha Linium. Les concentrations de Zn déterminées dans nos échantillons dénotent une contamination de la lagune de Korba.

Une concentration de $\mathrm{Pb}$ inférieure à $3 \mu \mathrm{g} / \mathrm{g}$ MS a été détectée dans un milieu non contaminé pour Enteromorpha intestinalis dans le golfe de San Jorge dans l'argentine (Muse et al., 1999). Lozano et al., 2003 ont considéré $10 \mu \mathrm{g} / \mathrm{g}$ MS de $\mathrm{Pb}$ la limite seuil entre un milieu contaminé ou non par le plomb.Nous pouvons dire que notre site dénote une contamination moyenne en plomb. L'accumulation de plomb dans les algues peut être expliquée par la présence d'un grand potentiel de fixation rapide et complète de $\mathrm{Pb}$ qui est absorbé à la surface membranaire vers le cytoplasme cellulaire de l'algue (Veselina et Stancheva, 2013).

La concentration de Mn dans notre site pour les deux macroalgues n’est pas élevée par rapport à des autres sites. Il n’existe pas une limite seuil du manganèse dans des sites non contaminés (Rybak et al., 2013;Al Homaidan et al., 2011). Mais d'autre auteurs expliquent l'accumulation du Mn dans les macroalgues par la nécessité de cet élément dans le processus de la photosynthèse II( Hall et RAO, 1999). Mn est nécessaire pour la libération de l'oxygène qui est utilisé par la majorité des organismes vivants pour leur respiration cellulaire.

Les concentrations en fer dans les algues vertes (Enteromorpha intestinalis et Chaetomorpha Linium) sont plus élevées que les autres éléments. Villares en 2001 a montré que l'accumulation du fer par Enteromorpha intestinalis est à son maximum en été (mai - juillet). Cependant, dans notre cas, Enteromorpha intestinalis accumule le fer au printemps et en hiver. De tel résultat peut être expliqué par l'augmentation de la teneur en Fe durant la saison pluviale que durant la saison sèche. L'une des origines du fer dans les cours d'eau est le lessivage du sol (Didier, 1995). La présence d'une forte concentration de Fe dans les plantes marines peut 
être attribuée au fait qu'ils sont des oligo-éléments importants pour les diverses fonctions métaboliques des plantes. Le fer joue un rôle important dans les processus de la respiration des algues (Wabbeh, 1984). Les concentrations de Fe dans la lagune de Korba sont généralement inférieures à celles détectées dans d'autres secteurs propres.
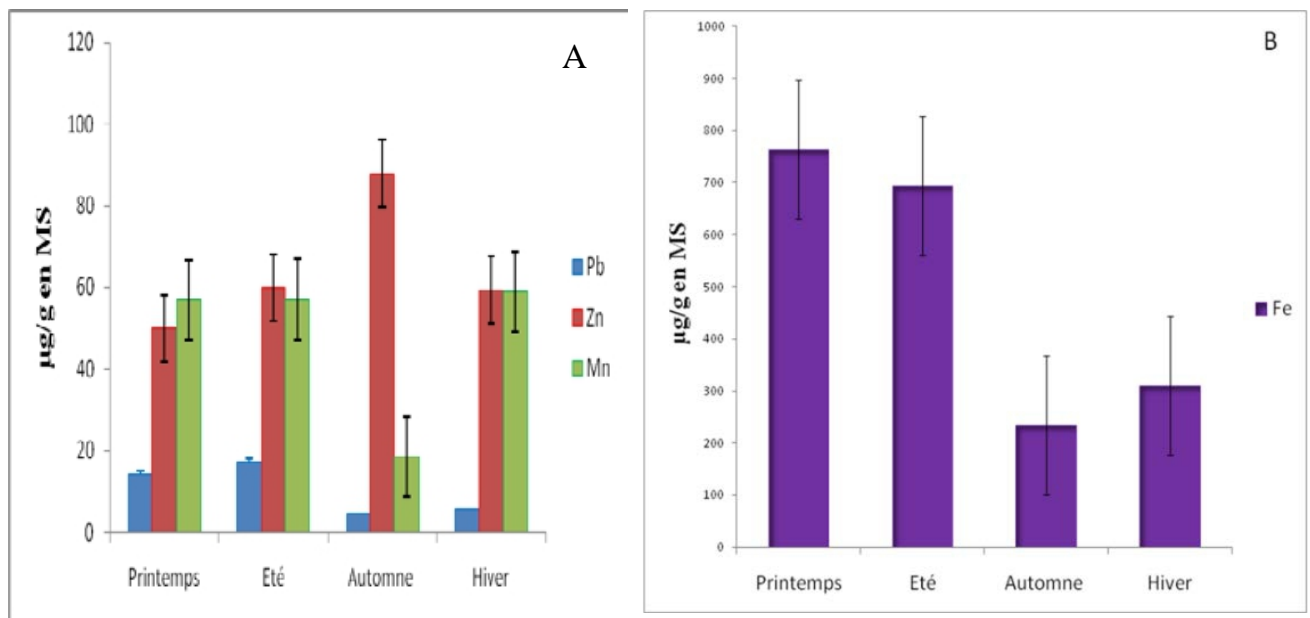

Figure 15 : (A; B) : Variation saisonnière en concentrations (en $\mu \mathrm{g} / \mathrm{MS}$ ) des métaux traces ( $\mathrm{Pb}, \mathrm{Zn}, \mathrm{Mn}$ et $\mathrm{Fe}$ ) dans Chaetomorpha Linium collectée de la lagune de Korba.
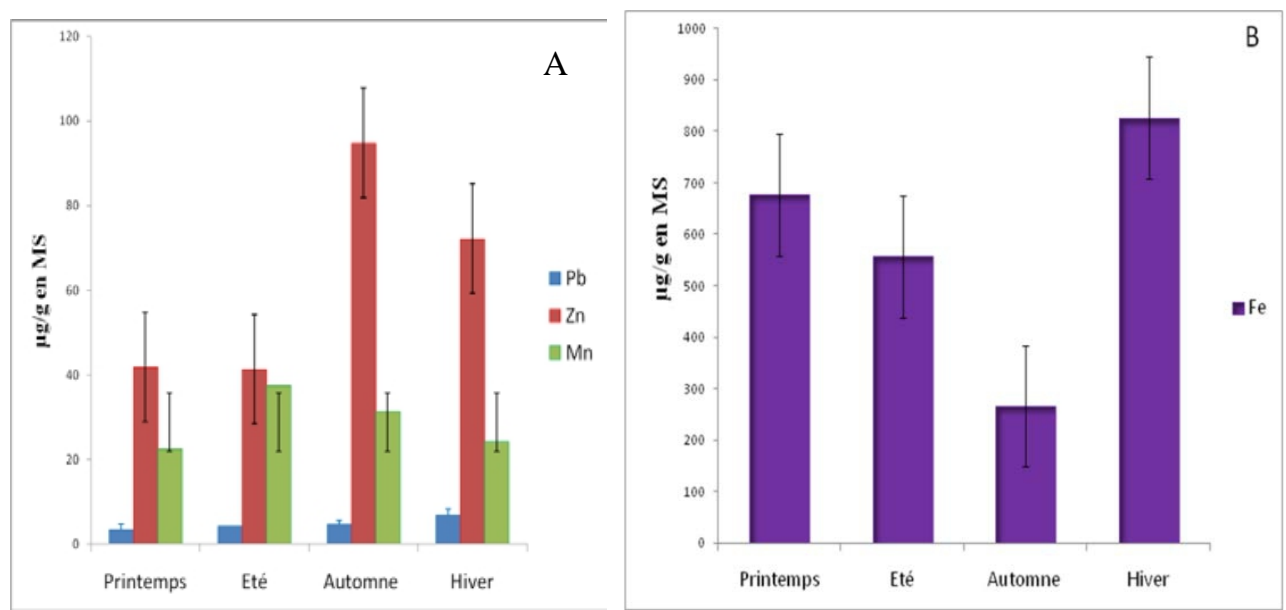

Figure 16(A et B) : Variation saisonnière des concentrations (en $\mu \mathrm{g} / \mathrm{MS}$ ) des métaux traces( $\mathrm{Pb}, \mathrm{Zn}, \mathrm{Mn}$ et $\mathrm{Fe})$ dans Enteromorpha intestinalis collectée de la lagune de Korba 

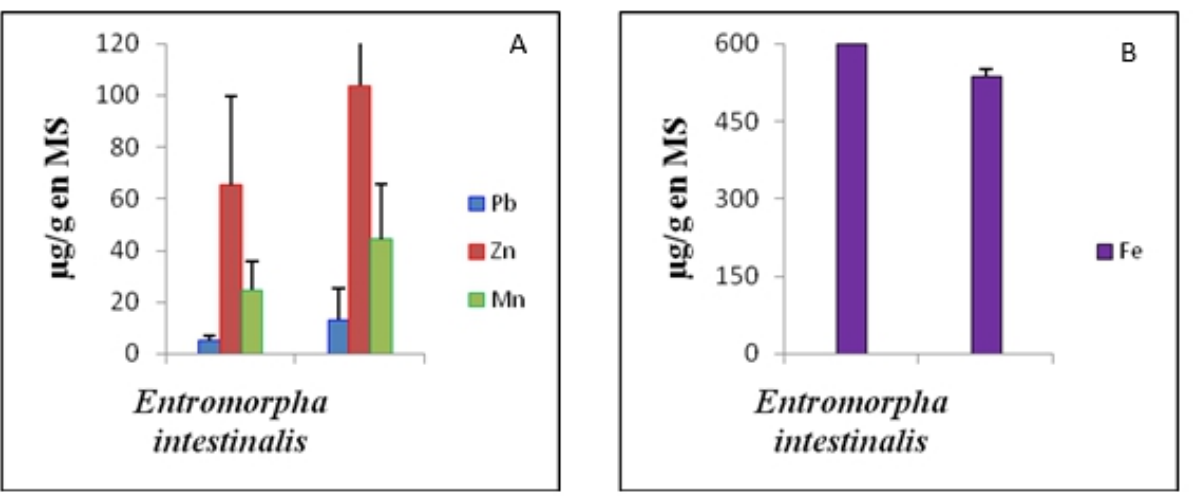

Figure 17 (A et B) : variation annuelle des concentrations des métaux traces ( $\mathrm{Pb}, \mathrm{Zn}, \mathrm{Mn}$ et Fe) chez les deux espèces collectées de la lagune de Korba.

\section{Conclusion}

La qualité deseaux de la lagune de Korba est variable en fonction des saisons. Cette variation a une grande influence sur la densité de la prolifération des algues vertes. Les concentrations élevées des paramètres physico-chimiques et l'apparition du bloom d'algues qui couvrent la surface de la lagune souligne l'eutrophisation de la lagune de Korba. L’étude de la teneur en métaux traces ( $\mathrm{Pb}, \mathrm{Fe}, \mathrm{Mn}$ et $\mathrm{Zn})$ chez les espèces d'algues étudiées (Enteromorpha intestinalis et Chaetomorpha linum) de la lagune de Korba montre que la bioaccumulation de ces métaux varie en fonction dela saison. C'est l'espèce Chaetomorpha linum qui a accumulé plus de métaux. Ainsi, le présent travail a mis en évidence l'importance de l'utilisation des macroalgues comme indicateurs biologiques de pollution métallique dans un écosystème.

\section{References:}

Akcali. I \&Kucuksezgin .F ,2011: A bio monitoring study: Heavy metals in macro-algae from Eastern Aegean coastal areas. Mar. Pollut. Bull. 62 : 637645.

Al-Homaidan A. A. , Al-Ghanayem A. , Alkhalifa A. H. , 2011 : Green algae as bioindicator of heavy metal pollution in Wadi Hanifah Stream, Riyadh, Saudi Arabia. Int. J. Water Resourc. Arid Environ. 1, 1: 10-15.

Barnett, B.E., Ashcroft, C.R. 1985. Heavy metals in Fucus vesiculosus in the Humber estuary, Environ. Pollut. Ser. B 9 : 193-213.

Bouden Salah, 2006:Caractérisation sédimentlogique et géochimique des sédiments de la lagune de Korba (cap bon, nord-est de la Tunisie). Thèse de doctorat. Université Tunis El Manar. p 270

Bouden Salah, Fredj Chaâbani et Saadi Abdeljaoued, 2009 : Dynamique sédimentaire de la lagune de Korba (Nord-Est de la Tunisie), Quaternaire, vol. 20/2 |, 227-237. 
Briand and all1989 : Prolifération de l'algue verte Ulva en Baie de Lannion ( France). Etude d'une nuisance and son traitement par fermentation anaérobie. Thèse 420 page.

Brown, M. T. , W. M. Hodgkinson and C. L. Hurd, 1999: Macroalgal diversity on accumulation of Spatial and temporal variations in the copper and zinc concentrations of two green seaweeds from Otago Harbour, New Zealand. Mar. Environ. Res. , 47: 175-184.

Chambers PA, Kent R, Charlton MN, Guy M, Gagnon C, Roberts E, Grove E, Foster N. 2001. Les éléments nutritifs et leurs effets sur l'environnement au Canada. Environnement Canada, p. 271.

Didier G,1995 la pollution des milieux aquatiques aide mémoire, 2 ème édition 220p.

Hedhli R, 1998 : Etude Biosedimentologique de la lagune de Korba DEA Université Tunis El Manar. p 65.

Heikskary S. , Markus H. 2001: Establishing relationships among nutrient concentrations, phytoplankton abundance, and biochemical oxygen demand in Minnesota, USA, rivers. Journal of Lake and Reservoir Management, 17: 251-267.

HALL D. O. , RAO K. K. , 1999: Photosynthesis. Cambridge University Press, Cambridge, UK.

Kaimoussi .Aziz , Abdelkrim Mouzdahir et Abdelkbir Saih ,2005 :Variations saisonnières des concentrations en métaux ( $\mathrm{Cd}, \mathrm{Cu}, \mathrm{Fe}, \mathrm{Mn}$ and $\mathrm{Zn}$ ) chez l'algue Fucus spiralis du littoral de la ville d'El Jadida (Maroc). Water Qual. Res. J. Canada, 2005. Volume40, No, 1, 102-110.

Lassaad CHOUBA, L. AJJABI CHEBIL et S. HERRY, 2010: Etude saisonnière de la contamination métallique des macroalgues de la lagune Nord de TUNIS.Bull. Inst. Natn. Scien. Tech. Mer de Salammbô, Vol. 37, 2010

Lawson E.O, 2011: Physico-Chemical Parameters and Heavy Metal Contents of Water from the Mangrove Swamps of Lagos Lagoon, Lagos, Nigeria, Advances in Biological Research, 5(1), 8-21.

LISEC 2004. : Contrôle van de physico chemische kwaliteit van de vis waters van het Brussels Hoofdstedelijk Gewest, avril 2004

Lozano, G., A. Hardisson, A.J. Gutierrez and M.A. Lafuente, 2003. Lead and cadmium levels in coastal benthic algae (seaweeds) of Tenerife, Canary Islands. Environ. Inter., 28: 627-631.

LUCIA M., BOCHER P., COSSON R.P., CHURLAUD C., BUSTAMANTE P. 2012: Evidence of species-specific detoxification processes for trace elements in shorebirds. Ecotoxicology, . 21(8): 2349-62.

Maanan.M, 2008 : Heavy metal concentrations in marine mollusks from the Moroccan coastal region. Environnemental Pollution 153, 176-183. 
Mohamed Amine HELALI 2015: Bilan de transfert des métaux lourds dans des milieux marins soumis aux apports d'origines naturelles et anthropiques :Cas du delta de l’Oued Medjerda Thèse de doctorat. Université Tunis El Manar. p 235

Muse, J.O., J.D. Stripeikis, F.M. Fernandez L. d'Hucique, M.B. Tudino, C.N. Carducci and O.E. Troccoli, 1999. Seaweeds in the assessment of heavy metal pollution in the Gulf San Jorge, Argentina. Environ. Pollut., 104: 315322.

Müller .M , Schirmer.M, Kettler.J,1993: Use of Enteromorpha intestinalis(Chlorophyceae) for active biomonitoring of heavy metals in the Weser estuary Netherland Journal of Aquatic Ecology June 1993, Volume 27, Issue 2, pp 189-195.

Oueslati W, 2011. Cycles biogéochimiques des métaux lourds dans les sédiments marins de la lagune de Ghar ElMelh. Thèse de doctorat. Université Tunis El Manar. p 271.

Phillips, D. J. H. , 1994: Macrophytes as biomonitors of trace metals. In: Kramer, K. J. M. (Ed),Biomonitoring of Coastal Waters and Estuaries. CRC Press Inc, Florida, pp. 85-103. heavy metal pollution Hydrobiologia 462: 221-232.

Rapin A, Blanc P, Corvec C. 1989. Influence des apports sur le stock de phosphore dans le lac Léman et sur l'eutrophisation. Revue Sciences de l'Eau, (2): 721-737.

Rodier J, Bazin C, Broutin JP, Chambon P, Champsaur H, Rodi L. 2005. The analysis of water: natural water, wastewater, seawater 8 ème édition. DUNOD. Paris, p. 1383

Rybak A. , Messayaz B. , Pikosz M. , Szendzina L. , Łęska B. , 2013. Accumulation of heavy metals (Co, Cr, Cu, Mn, Zn) in the freshwater alga Ulva type, sediments and water of the Wielkopolska region, Poland. NaukaPrzyr. Technol. 7, 4.

Sawidis .T, Brown .M.T, Zachariadis.G and Stratis.I, , 2001. Trace metal concentrations in marine macroalgae from different biotopes in the Aegean Sea. Environ. Inter. , 27: 43-47.

Say, P. J. , J. G. Burrows \& B. A. Whitton, 1990 : Enteromorpha as a monitor of heavy metals in estuaries. Hydrobiologia 195: 119-126.

Strickland, J. D. H. and T. R. Parsons, 1972: A Practical Handbook of Seawater Analysis. 2nd ed., Bull.Fish.Res. Bd. Can. No. 167, 310 pp.

Veselina Panayotova, Mona Stancheva , 2013 : Mineral composition of marine macroalgae from the Bulgarian Black Sea Coast Scripta Scientifica Medica, vol. 45, suppl. 6, 2013, pp. 42-45 Copyright Medical University of Varna.

Villares . R, Puente. X \&Carballeira. A ,2001: Ulva and Enteromorpha as indicators of heavy metal pollution Hydrobiologia 462: 221-232. 
Villares. R, Puente . X, Carballeira .A, 2002 .Seasonal variation and background levels of heavy metals in two green seaweeds. Environ. Pollut. 119:79-90.

Wabbeh, M. , 1984. Levels of Zn, Mn, Mg, Fe and Cd in three species of sea grasses from Agaba (Jordan). Aquatic Botany, 20: 179-183. 